\title{
The investigation of regional climate anomalies with a linear barotropic model and an adjoint technique
}

\author{
By ANDREW W. ROBERTSON ${ }^{1}$ \\ Meteorological Institute, University of Munich, Theresienstr. 37, 8000 Munich 2, Germany
}

(Received 17 December 1991; revised 18 May 1992)

\section{SUMMARY}

The adjoint of a steady-state linear barotropic model is presented as a tool for diagnosing regional upperlevel vorticity anomalies occurring in general circulation model (GCM) experiments. As a starting point, a simulation of a chosen GCM vorticity anomaly is made using a standard linear barotropic model forced by the corresponding anomalies in the GCM's upper-level divergence and transient-forcing fields. The associated adjoint model is then used to interpret this linear simulation over a chosen geographical region, in terms of the linear model's global forcing field. To do this, the adjoint model is forced by a local information source situated over the chosen region. The resulting adjoint solution consists of a global spatial weighting function which can then be used to identify regions of the forcing-local or remote-associated with the regional anomaly. The validity of barotropic linear theory can also be quantified as a by-product.

As an illustration, the model is applied to a large interdecadal anomaly over Europe, arising in a 100-year doubled $\mathrm{CO}_{2}$ integration of the Hamburg coupled ocean-atmosphere GCM. Linear barotropic theory is found to account for over $60 \%$ of the European interdecadal anomaly amplitude in terms of anomalous divergence and the effects of transients. Although a large part of the effects of anomalous divergence are spatially concurrent with the European anomaly and just upstream of it, remote forcing regions over the east Pacific and tropical Atlantic are also implicated. Anomalous transients are found to play a secondary role. The linear results are compared with some one-point correlation maps of the GCM's interdecadal variability.

\section{INTRODUCTION}

In a previous article (Robertson 1992b), the author used the adjoint of a one-layer tracer model to investigate the importance of anomalous temperature advection in accounting for a 10-January tropospheric temperature anomaly over central Europe in a doubled $\mathrm{CO}_{2}$ general circulation model (GCM) experiment. It was found that, compared to the direct effects of anomalous diabatic heat sources and sinks, the effect of changes in wind direction in accounting for the anomaly was overwhelming. It was suggested that anomalous equivalent barotropic stationary Rossby waves might have been associated with the anomalous advection pattern, and that barotropic linear theory might be used to trace the roots of the associated vorticity anomaly using the adjoint of a steady-state barotropic vorticity-equation model. The purpose of the present paper is to follow up this suggestion.

Held and Kang (1987) have shown that a steady-state barotropic vorticity-equation model linearized about a zonally-asymmetric $300 \mathrm{mb}$ time-mean basic flow, can be remarkably successful at capturing the zonally asymmetric part of the extratropical response to $\mathrm{El} \mathrm{Niño,} \mathrm{if} \mathrm{divergence} \mathrm{and} \mathrm{vorticity} \mathrm{tendencies} \mathrm{due} \mathrm{to} \mathrm{transients} \mathrm{are}$ prescribed. There is reason to suppose that steady-state linear barotropic theory might also illuminate extratropical anomalies on interdecadal timescales.

A leading theory proposes that climate variability on interdecadal timescales is driven by subsystems of the climate system whose dynamics have timescales of this order; notably the upper ocean, which integrates in time the effects of high-frequency whitenoise atmospheric motions (Hasselmann 1976; Mikolajewicz and Maier-Reimer 1990). Low-frequency components of the atmosphere are seen as a response to anomalous external (oceanic) forcing, although atmospheric feedback on the ocean may be crucial

\footnotetext{
${ }^{1}$ Present affiliation and correspondence address: Department of Atmospheric Sciences, University of California, 405 Hilgard Avenue, Los Angeles, CA 90024-1565, U.S.A.
} 
to the dynamics of the coupled system. A corollary of this theory is that decadal flow anomalies over central Europe might have their ultimate roots in remote sea surface temperature (s.s.t.) anomalies. If this were to be the case, the atmosphere with its much shorter characteristic timescales could be approximated by a steady-state model. Furthermore, motivated by the results of Held and Kang, a steady-state linear barotropic model might make a useful tool for investigating the remote atmospheric response to oceanic forcing, with the latter expressed in terms of upper-level divergence and transient forcing anomalies. An important aspect of the linear barotropic model is the implicit inclusion of certain nonlinear effects in the divergence and transient forcing terms; a sword which cuts both ways with success in simulation being offset by the prescription of important internal dynamical effects.

The regional problem can be tackled by applying the adjoint method of Marchuk (1974) to the linear steady-state barotropic model. This adjoint method allows the linear model's regional response to be expressed in terms of global distributions of the prescribed forcing (i.e. divergence and vorticity tendencies due to transients) via a spatial weighting function. In addition, the weighted 'residual forcing' required to make the linear simulation exactly fit the GCM regional anomaly, can give a measure of the validity of the linear model together with its sensitivity to parameters. The promise of the adjoint method for tracing the roots of regional anomalies was demonstrated by Robertson $(1992 \mathrm{a}, \mathrm{b})$; but the method's usefulness rests on the relevance of the linear model to which it is applied.

In this paper we illustrate the use of the adjoint of a linear barotropic model to examine 10-winter $300 \mathrm{mb}$ vorticity anomalies over central Europe occurring in a 100 year doubled $\mathrm{CO}_{2}$ response experiment (referred to as $2 \times \mathrm{CO}_{2}$ in the following) and a control run of the Hamburg coupled GCM. Two sample decades are considered: decade A corresponds to a modest negative vorticity anomaly over central Europe, and decade $\mathrm{B}$ is the case investigated by Robertson (1992b). Anomalies are defined as differences between 10-winter episodes; either subtracting the control from the $2 \times \mathrm{CO}_{2}$ run (the interdecadal $\mathrm{CO}_{2}$ 'signal'), or considering the control or $2 \times \mathrm{CO}_{2}$ runs separately (the pure interdecadal variability). The GCM experiments are outlined in section 2 and discussed in terms of central European upper-level vorticity anomalies. In section 3 , we develop the theory of the adjoint method applied to the barotropic model. The results of applying the method are presented in section 4 and we end with a discussion in section 5 .

\section{THE GCM EXPERIMENTS}

Our data-base consists of two 100-year GCM experiments performed with the Hamburg climate model; a doubled $\mathrm{CO}_{2}$ run (where the effective $\mathrm{CO}_{2}$-concentration was instantaneously doubled and then held fixed) together with a control run. A description of both the model and the experiments can be found in Cubasch et al. (1991). The model consists of a 19-level atmospheric GCM truncated triangularly at total wavenumber $\mathrm{n}=21$ (T21) coupled to an 11-level ocean model of similar horizontal resolution. The ocean model is based on a formulation of the primitive equations appropriate for largescale geostrophic motion. Coupling is synchronous, and a flux correction is applied to eliminate climate drift.

Figure 1 illustrates 99-winter (December, January, February-DJF) time series of the vorticity at $300 \mathrm{mb}$ averaged over central Europe for both GCM runs. The central European average is computed using the spatial window-function illustrated in Fig. 2, 


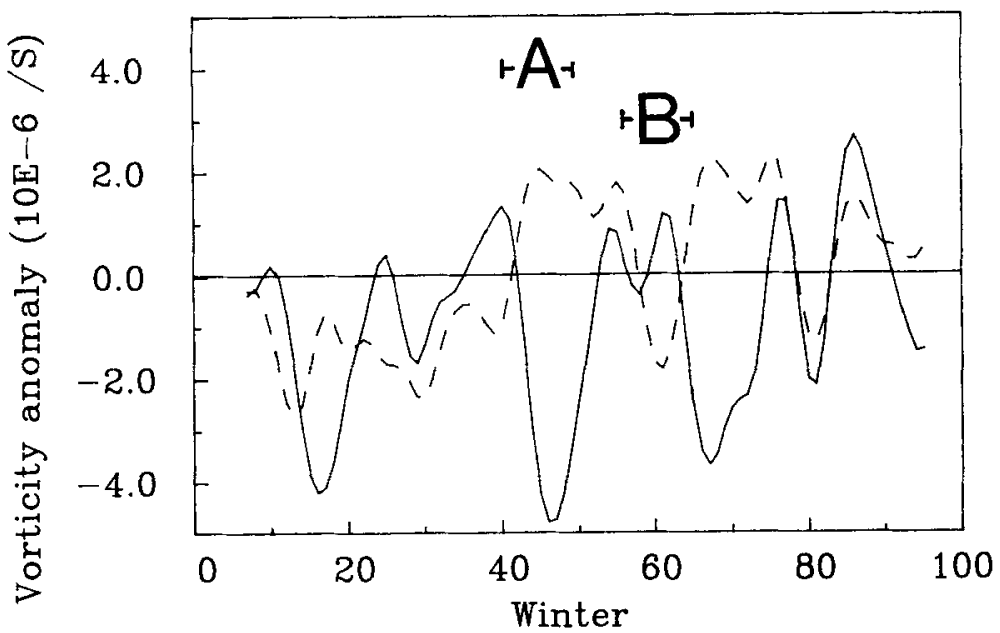

Figure 1. Low-pass filtered time series of $\bar{\zeta}^{G}$ ( $300 \mathrm{mb}$ DJF mean, $G=$ central Europe) for control (dashed) and $2 \times \mathrm{CO}_{2}$ (solid line) GCM experiments. Curves are plotted relative to the 99 -winter mean of $\xi^{G}$ in the control experiment $\left(7.63 \times 10^{-6} \mathrm{~s}^{-1}\right)$. The filter (11-point Gauss) retains periods greater than about 10 winters. The standard deviations of filtered time series are $1.30 \times 10^{-6} \mathrm{~s}^{-1}$ (control) and $1.46 \times 10^{-6} \mathrm{~s}^{-1}\left(2 \times \mathrm{CO}_{2}\right)$. The sample decades $A$ and $B$ are indicated (December, year 39-February, year 49 and December, year 55February, year 65 , respectively).

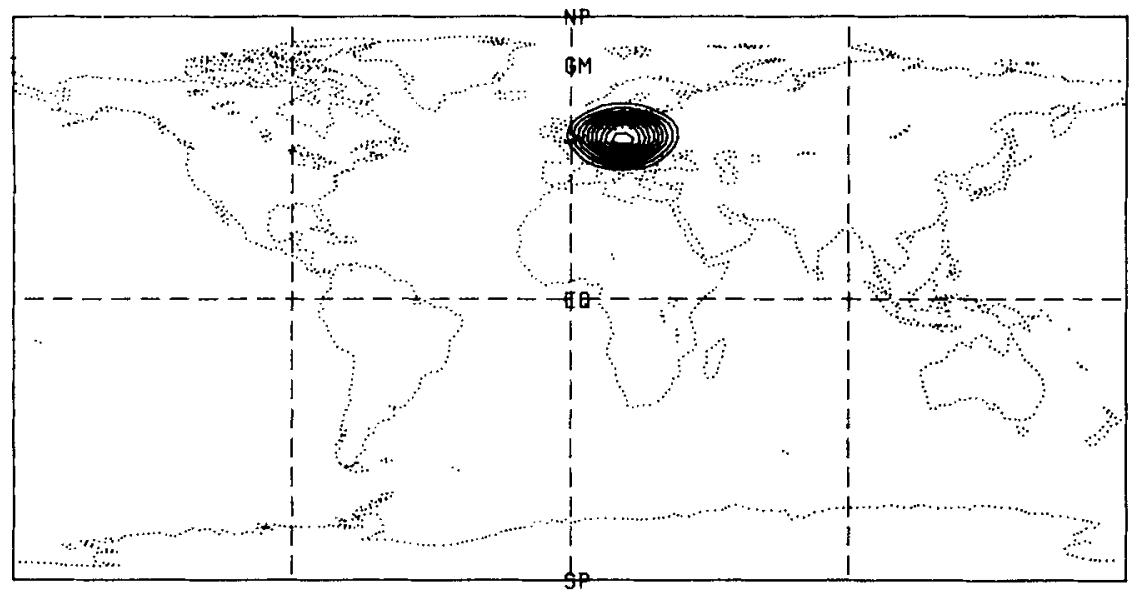

Figure 2. Window function or conjugate forcing function, $F^{*}$, situated over central Europe and truncated at T21 (hence the rounded form). Contour interval $25 A^{-1}$ where $A$ is the area of the sphere; zero contour omitted. The Greenwich meridian is at the centre of the plot and all other cylindrical plots.

and the time series has been low-pass filtered to select periods greater than about 10 winters. Both curves exhibit a pronounced interdecadal variability and there is very little mean $\mathrm{CO}_{2}$ 'signal': anomalies being plotted with respect to the 99-winter mean of the control run. The size of the interdecadal variance in the control run underlines the importance of purely internal variability of the coupled system for regional climate on interdecadal timescales, whilst there is only a small and statistically insignificant increase in variance with doubled $\mathrm{CO}_{2}$. 


\section{THE ADJOINT MODEL}

Any steady-state linear model may be written

$$
\mathrm{L} \zeta=F
$$

where $L$ represents the differential operator linearized about some basic state, together with the boundary conditions of the problem. Here $\zeta$ is the solution obtained when the forcing $F$ is specified. Equation (1) constitutes the usual direct linear forced problem. Now the linear operator $L$ is associated with an adjoint operator $L^{*}$, which is defined by Green's identity:

$$
\langle\eta, \mathrm{L} \zeta\rangle=\left\langle\mathrm{L}^{*} \eta, \zeta\right\rangle
$$

where $\eta$ is some other solution field and $\langle.,$.$\rangle denotes the inner product, whose choice$ affects $L^{*}$. We can construct a problem similar to (1) for the adjoint operator, with some arbitrary forcing function $F^{*}$, whose solution is $\zeta^{*}$ :

$$
\mathrm{L}^{*} \zeta^{*}=F^{*}
$$

and this constitutes the adjoint problem corresponding to (1). Then, inspired by (2), we can form the inner product of the terms in (1) with $\xi^{*}$, the inner product of the terms in (3) with $\zeta$, and subtract, yielding

$$
0=\left\langle\zeta^{*}, F\right\rangle-\left\langle\zeta, F^{*}\right\rangle
$$

The left-hand side vanishes by definition (2) so that any non-orthogonality between the linear-model solution and $F^{*}$ is expressed in terms of the non-orthogonality between the linear-model forcing and the adjoint solution.

In order to put (4) to use, the inner product must have a physical sense. If we define it in terms of a scalar product in physical space, then $F^{*}$ and $\zeta^{*}$ can be interpreted as spatial weighting functions of $\zeta$ and $F$ respectively. Thus we define

$$
\left\langle\zeta, F^{*}\right\rangle \equiv \frac{1}{4 \pi} \int_{-1}^{+1} \int_{0}^{2 \pi} \zeta F^{*} \mathrm{~d} \lambda \mathrm{d} \mu
$$

where $\lambda$ denotes longitude and $\mu=\sin$ (latitude). Equation (5) corresponds to the inner product of two vorticity fields under an enstrophy norm, although the inner products in (4) are not enstrophies as will become clear. We define $F^{*}$ to be a local weight by giving it unit amplitude in a chosen region $G$ and zero amplitude elsewhere:

$$
\begin{aligned}
F^{*} & =4 \pi /|G| & & \text { for }(\lambda, \mu) \in G \\
& =0 & & \text { elsewhere }
\end{aligned}
$$

as illustrated in Fig. 2. In this case (5) yields as desired

$$
\bar{\zeta}^{G}=\left\langle\xi, F^{*}\right\rangle
$$

where $\bar{\xi}^{G}$ is the average vorticity in region $G$. Finally rewriting (4) using (5) and (7), we arrive at the expression

$$
\bar{\zeta}^{G}=\frac{1}{4 \pi} \int_{-1}^{+1} \int_{0}^{2 \pi} F \zeta^{*} \mathrm{~d} \lambda \mathrm{d} \mu
$$

so that the linear model solution averaged over $G$ is given by the global integral of its forcing weighted by the global solution of the locally-forced adjoint problem. Note that $\zeta^{*}$ has the dimensions $\mathrm{m}^{-2}$; so it is not a vorticity. This 'adjoint method' was first derived 
in a meteorological context by Marchuk (1974) and its application as a climate model diagnostic was first suggested in 1990 by Joseph Egger (personal communication).

In the case of the barotropic vorticity equation in pressure coordinates, (1) can be written in the form

$$
\overline{\mathbf{v}}_{\psi} \cdot \nabla \zeta+\mathbf{v}_{\psi} \cdot \nabla(\bar{\zeta}+f)-{ }_{K} \nabla^{2} \zeta+\alpha \zeta=F
$$

where the overbar denotes a basic state variable and unbarred variables represent anomalies. Here $\mathbf{v}_{\psi}$ is the rotational wind, $f$ is the Coriolis parameter, $\zeta$ is, from now on, the relative vorticity, and all variables are time averages. With a view to numerical solution, friction is represented in the usual manner by a linear damping (with coefficient $\alpha$ ) plus a small harmonic diffusion (with coefficient $K$ ). If the basic state is chosen to be some decadal-average flow, then the inhomogeneous 'forcing' may be expressed as

$$
F=-\nabla \cdot\left(\mathbf{v}_{\chi}(\bar{\zeta}+f)\right)+T+C
$$

where $\mathbf{v}_{\chi}$ denotes the anomalous divergent wind, $T=-\nabla \cdot\left(\mathbf{v}^{\prime} \zeta^{\prime}\right)$ is the vorticity tendency due to anomalous sub-decadal transients and $C$ includes all the nonlinear, baroclinic and other terms in the GCM's vorticity equation which were omitted from (9), i.e.

$$
C=-\nabla \cdot(\mathbf{v} \xi)-\mathbf{k} \cdot \nabla \times\left(\omega \frac{\partial \mathbf{v}}{\partial p}+\bar{\omega} \frac{\partial \mathbf{v}}{\partial p}+\omega \frac{\partial \overline{\mathbf{v}}}{\partial p}\right)+\alpha \zeta+B
$$

where $\mathbf{k}$ is the unit vector in the vertical direction and $B$ denotes the GCM's subgridscale parametrizations, excluding horizontal diffusion, together with interpolation errors in transferring from the GCM's hybrid vertical coordinates to pressure coordinates. Note that the linear damping term which we included in the linear operator on the left-hand side of (9) has no counterpart in the GCM so that it reappears in $C$, whilst the diffusion term may be chosen to be that used in the GCM so that it occurs only in the linear operator.

Using integration by parts it may be shown that the adjoint equation (3) associated with (9a) takes the form

$$
-\overline{\mathbf{v}}_{\psi} \cdot \nabla \zeta^{*}-\Delta^{-1} \nabla \cdot\left\{(\bar{\zeta}+f) \mathbf{k} \times \nabla \zeta^{*}\right\}-\kappa \nabla^{2} \zeta^{*}+\alpha \zeta^{*}=F^{*}
$$

where $\Delta \equiv \nabla^{2}$. Substituting (9b) into (8) we obtain

$$
\bar{\zeta}^{G}=\frac{1}{4 \pi} \int_{-1}^{+1} \int_{0}^{2 \pi}(R+T+C) \xi^{*} \mathrm{~d} \lambda \mathrm{d} \mu,
$$

where $R \equiv-\nabla \cdot\left(\mathbf{v}_{\chi}(\bar{\zeta}+f)\right)$ is the so-called Rossby wave source.

The problem is discretized in terms of spherical harmonics and the matrix corresponding to the linear operator in (1) is constructed using the transform method (e.g. Hoskins and Karoly 1981). Equation (12) is solved by firstly calculating $\xi^{*}$; but rather than using (11) directly, it is more convenient to use (3) because the adjoint of a matrix operator is very easily constructed. In the Appendix it is shown that the adjoint is simply the transpose matrix of the linear operator, modified according to the definition of the inner product. The discretized form of the inner product in (12) is also given in the Appendix.

In the following section we present solutions of the linear model (9) linearizing about decadal-mean basic flows taken from the GCM. The adjoint method (12) is then used to decompose these linear solutions over central Europe. The basic states are generally taken to be wavy (i.e. they have spatial variation in both latitudinal and longitudinal directions) and we consider the zonally-asymmetric part of the response. The $300 \mathrm{mb}$ 
level is used throughout. Unless otherwise stated, a 5-day linear damping is included in the linear operator, together with the diffusion configuration of the GCM. The heavy damping proves to be necessary to control the near resonance of the linear operator, whilst sensitivity to the diffusion is small. The transient forcing $T$ is computed explicitly using daily data, including all periods less than a decade and including the divergent component of the wind.

\section{REsults}

The linear model and its adjoint are now applied to three interdecadal $300 \mathrm{mb}$ vorticity anomalies over the region $G$ comprising central Europe (Fig. 2).

\section{(a) Case 1}

We consider first the $\mathrm{CO}_{2}$ 'signal' defined as

$$
\zeta_{\mathrm{a}}=\zeta_{\mathrm{r}}-\zeta_{\mathrm{c}}
$$

where $\zeta_{\mathrm{r}}$ denotes a 10-winter (DJF) average of the response GCM experiment $\left(2 \times \mathrm{CO}_{2}\right)$ over decade A (cf. Fig. 1) and $\zeta_{\mathrm{c}}$ the corresponding average of the control run. This
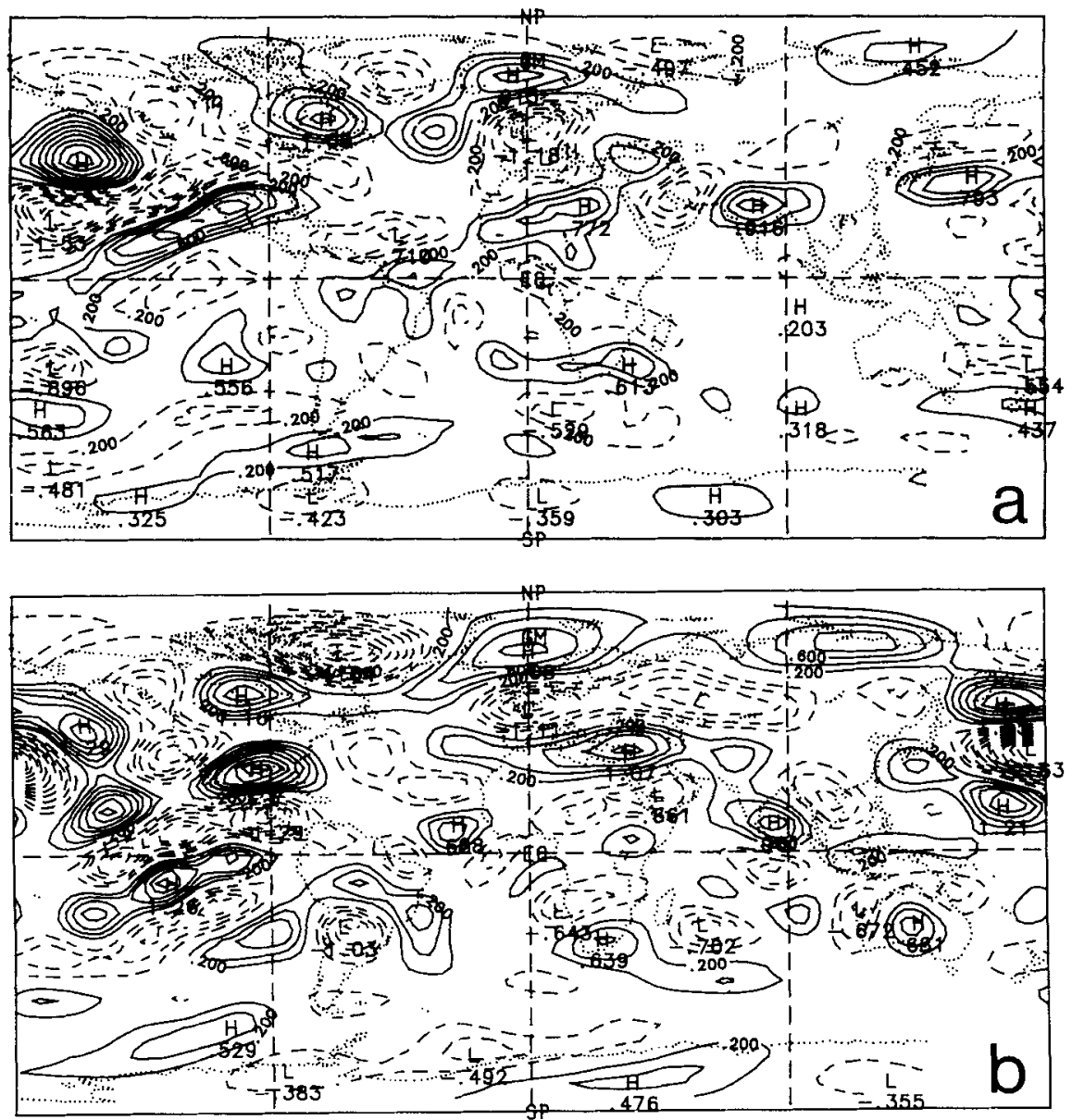

Figure 3. Case I: (a) 10-winter mean $300 \mathrm{mb}$ vorticity anomaly $2 \times \mathrm{CO}_{2}-$ control for decade A (zonal mean removed). (b) Linear model simulation. Contour interval is $0.2 \times 10^{-5} \mathrm{~s}^{-1}$; zero contour omitted. 
particular decade is characterized over central Europe by a modest negative vorticity anomaly at $300 \mathrm{mb}$. Figure 3(a) illustrates the spatial distribution of $\zeta_{\mathrm{a}}$, and Fig. 3(b) illustrates its linear simulation by linearizing $(9)$ about $\zeta_{\mathrm{c}}$ and forcing with $F_{\mathrm{a}}$. For linear theory to be valid, we require $\left|\zeta_{a}\right| \ll\left|\zeta_{c}\right|$, which is certainly well satisfied (zonally asymmetric parts are implied throughout in the following). We set $C=0$ in (9b) so that this is just the familiar forced linear problem. The success of the linear simulation in Fig. 3 (b) varies considerably from place to place. It is rather poor over the Pacific and North America but qualitatively correct over Europe, predicting an anticyclone sandwiched meridionally between cyclonic flow. The wavetrain-like structure arcing from the east Pacific over Greenland into Europe is qualitatively reproduced. The linear damping $\left(\alpha^{-1}=5 \mathrm{~d}\right)$ was chosen to give a reasonable solution amplitude, but nevertheless, nearresonant structures become a problem at high latitudes; while decreasing the damping strength brings the simulation hopelessly close to resonance. We note that although eigenanalysis reveals that the fastest-growing mode has an e-folding time of 13 days, with a period of 20 days (W. Metz, personal communication), near-resonance can still be a problem with damping times of 5 to 10 days. This is an inherent problem with this type of forced linear model, as was discussed by Branstator (1992). Although the success of the linear model over Europe may be fortuitous, local nonlinear processes elsewhere may be responsible for the poor simulation in many locations.

Maps of the components of $F_{\mathrm{a}}$ are displayed in Fig. 4 as vorticity tendencies. The anomalous Rossby wave source (Fig. 4(a)) has a wavetrain-like structure extending over the North Atlantic and Europe, and significant features over the east and north Pacific. The pattern is largely confined to the northern hemisphere. It is tempting to associate the wavetrain in $\zeta_{\mathrm{a}}$ (Fig. 3) from the east Pacific to Europe with upstream Rossby wave sources over the Pacific, and in situ forcing over the North Atlantic and Europe. Vorticity tendencies associated with sub-decadal transients (Fig. 4(b)) are characterized by smaller spatial scales and maxima in the northern hemisphere over the east Pacific, the east Atlantic and over Japan. Figure 4(c) illustrates the term $C$ from (10), computed as a residual from (9), which constitutes the additional forcing which one would need to add in order to obtain a perfect global linear solution. The damping contribution to $C$ (viz. (10)) is in fact scarcely visible in Fig. 4(c), which presumably mainly reflects baroclinic effects since the nonlinear term (not shown) becomes comparable only over the east Pacific. Nevertheless in order to produce a perfect solution, it is crucial that the damping contribution be included in $C$ because the linear model is very close to resonance. Indeed it turns out that if the latter is omitted from $C$, the resulting global solution is rather similar to that in Fig. 3(b), where $C$ was not included at all.

It is not obvious from Fig. 3 whether it is possible to interpret the anomalous anticyclone over Europe in terms of barotropic linear theory or not. Furthermore, a rather uncertain trial-and-error process would be required to assess which aspects of the forcing in Fig. 4 contribute to the European part of the solution, and to quantify the effects of the correction term. It is here that the adjoint method is effective. The top row of Table 1 shows the values of the global integrals in (12), computing $\xi^{*}$ via (3) and (A5), using the same damping and diffusion in the adjoint operator and including zonal asymmetries in the basic state. About $43 \%$ of the modest central European vorticity anomaly $\left(\bar{\zeta}_{\mathrm{a}}^{G}=-4.4 \times 10^{-6} \mathrm{~s}^{-1}\right)$ is accounted for by anomalous Rossby wave sources (RWS), $19 \%$ by anomalous transients, and the remaining $38 \%$ by the correction term. Thus barotropic linear theory accounts for over $60 \%$ of the central European vorticity anomaly in terms of anomalous divergence and transients.

The spatial distribution of the adjoint weighting function $\zeta^{*}$ can be used to identify which geographical features of $R_{\mathrm{a}}$ and $T_{\mathrm{a}}$ in Fig. 4 play a role in accounting for the central 

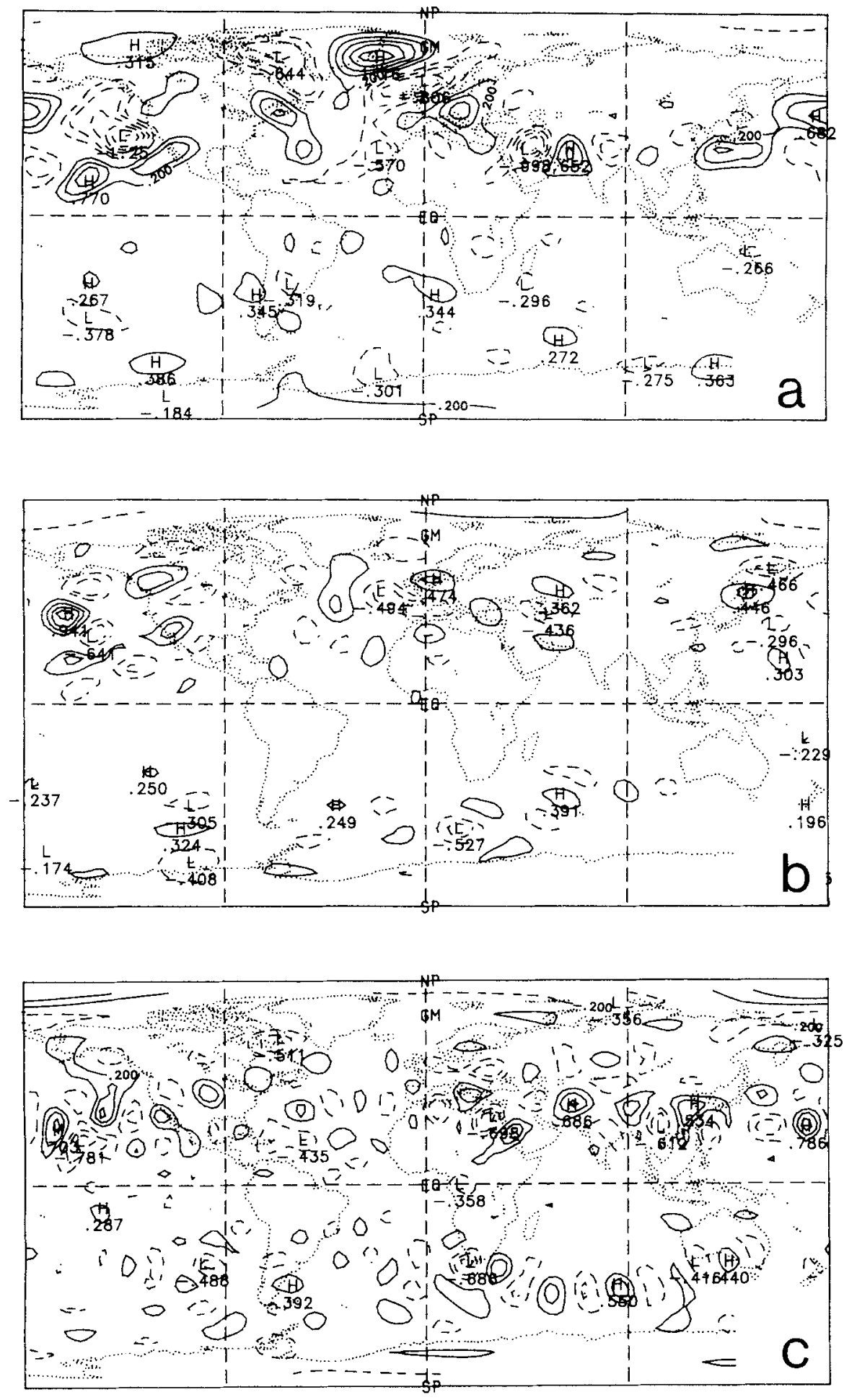

Figure 4. Components of the linear model forcing in Case I: (a) Rossby wave source $R_{\mathrm{d}}$; (b) vorticity tendency due to anomalous transients $T_{i a}$; (c) correction term $C$ needed to perfectly simulate Fig. 3(a). All ficlds are vorticity tendencies with zonal mean subtracted and contour interval $0.2 \times 10^{-5} \mathrm{~s}^{-1} \mathrm{~d}^{-1}$; zero contour omitted. 
TABLE 1. THE GLOBAL INTEGRALS IN (12) FOR CASE I

\begin{tabular}{lcccc}
\hline Experiment & $\begin{array}{c}\text { Vorticity anomaly } \\
\bar{\zeta}_{\mathrm{a}}\end{array}$ & $\begin{array}{c}\text { RWS } \\
\int R_{\mathrm{a}} \zeta^{*}\end{array}$ & $\begin{array}{c}\text { Transients } \\
\int T_{\mathrm{a}} \zeta^{*}\end{array}$ & $\begin{array}{c}\text { Correction } \\
\int C \zeta^{*}\left(\int \alpha \zeta \zeta^{*}\right)\end{array}$ \\
\hline WAVY & -4.44 & -1.89 & -0.88 & $-1.67(-0.99)$ \\
SYMM & & -4.00 & +1.34 & $-1.79(-3.31)$ \\
DAMP/2 & & +0.88 & -1.76 & $-3.56(-1.32)$ \\
DAMP*2 $_{\zeta}=\zeta_{r}$ & & -1.73 & +0.81 & $-3.53(-3.08)$ \\
\hline
\end{tabular}

Key: WAVY, wavy control basic state; SYMM, zonally symmetric basic state; DAMP/2, wavy basic state with damping halved to $\left(10 \mathrm{~d}^{-1}{ }^{-1}\right.$; DAMP*2, wavy basic state with damping doubled to $(2.5 \mathrm{~d})^{-1} ; \bar{\xi}=\zeta_{5}$, as WAVY but linearizing about $2 \times \mathrm{CO}_{2} 10$-winter mean. The column on the right gives the linear damping contribution to the correction integral. Units are $10^{-6} \mathrm{~s}^{-1}$.

European anomaly. Figure 5 illustrates $\zeta^{*}$ plotted on the northern hemisphere using a polar stereographic project ( $\zeta^{*}$ scarcely penetrates into the southern hemisphere). The adjoint response to a local conjugate forcing over central Europe is a mixture of wavetrain-like structures emanating out of the target region where the response is strongest, together with (adjoint) normal-mode-type structures. The latter dominate south of latitude $30^{\circ} \mathrm{N}$ and strongly resemble the adjoints of the fastest-growing normal modes of the barotropic vorticity equation computed by Zhang (1988) who plotted the streamfunction pattern $\left(\Delta^{-1} \zeta^{*}\right)$ associated with adjoint modes computed under an energy norm, which is equivalent to plotting the adjoint modes themselves under an enstrophy norm, as in our Fig. 5. The wavetrain extending over Greenland and perhaps as far as the east Pacific appears to have a counterpart in the linear forced problem in Fig. 3(b),

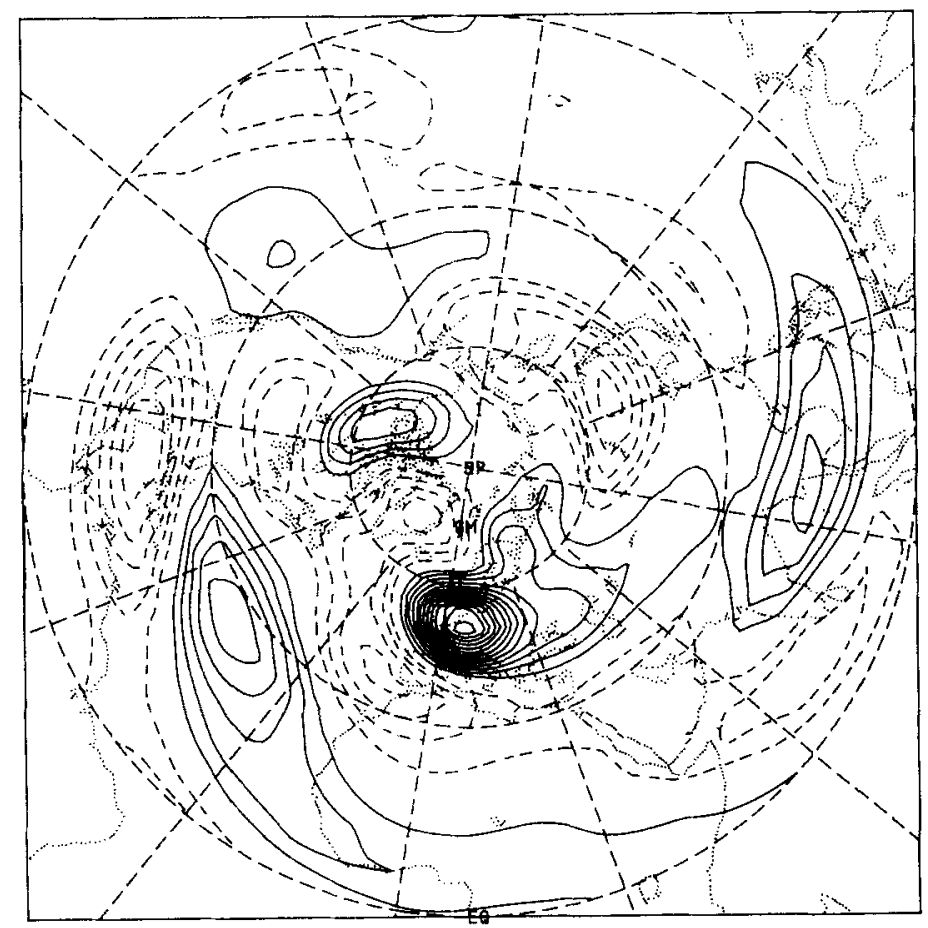

Figure 5. Adjoint solution $\zeta^{*}$ for Case I. Contour interval $25 \mathrm{~s} A^{-1}$; zero contour omitted. All such polar stereographic projections have the Greenwich meridian at bottom left centre. 
and there is a hint of a second wavetrain propagating 'backward' to the south-west of central Europe.

The wave-like character of $\zeta^{*}$ contrasts with the single-signed (positive) adjoint variable computed by Robertson $(1992 a, b)$ from the adjoint of a time-dependent tracer equation for temperature advection, where the evolution of the wind field was prescribed. In that case the adjoint variable was interpreted in terms of backward trajectories of a passively advected tracer. By contrast $\xi^{*}$ might be thought of as a tracer of Rossby wave propagation into a particular region, where the relative phases of $\zeta^{*}$ and the forcing determine the phase of the Rossby wave impinging on the target region.

The global integrals in Table 1 which account for $\bar{\zeta}_{a}^{G}$ may be interpreted in terms of the anomalous forcing distributions in Fig. 4, weighted by $\zeta^{*}$. Dynamically, $\left|\zeta^{*}\right|$ singles out regions which efficiently excite global normal modes with large amplitude over the target region, and regions which can excite local Rossby wave propagation towards Europe. The local sign of $\zeta^{*}$ determines the phase of the respective modes over the target region. For both regional energy propagation and global modes, zonal asymmetries in the basic state are crucial; as will be seen below. The weighted forcing distributions are illustrated in Fig. 6, again plotted polar stereographically, since it is the area integrals that are summed in (12).

Although the choice of inner-product norm affects the adjoint operator and thus also the spatial distribution of $\zeta^{*}$, the products in (12) and Table 1 (and their spatial distributions) are independent of the choice of the norm, because they involve both the adjoint variable and the inner product, as is demonstrated in the Appendix.

The weighted Rossby wave source (Fig. 6(a)) has its maximum over central Europe itself, with large magnitudes immediately upstream and over Greenland. This pattern is associated with large amplitudes in both $\xi^{*}$ and $R_{\mathrm{a}}$ and it is no accident that both have wavetrain-like structures upstream of central Europe. The wavetrain in the direct linear solution impinges on central Europe and thus has a counterpart in $\zeta^{*}$. In addition, the Rossby wavetrain in the direct linear forced problem has a divergence structure associated with it, and this is particularly pronounced owing to the orography of Greenland. We note that the wavelike structure of the Greenland wavetrain in $R_{\mathrm{a}} \zeta^{*}$ indicates that the associated wavetrains in $R_{\mathrm{a}}$ and $\zeta^{*}$ interfere with each other, with the latter having a larger wavelength than the former.

The adjoint solution $\zeta^{*}$ naturally has its largest amplitude over the conjugate forcing region, so that the local anomaly is most efficiently excited by local forcing. However, because the upstream pattern of $R_{\mathrm{a}} \zeta^{*}$ is wavelike there is a great deal of spatial cancellation and it is difficult to ascertain even the sign of the sum of these local and upstream effects. Furthermore, the pattern of $R_{\mathrm{a}}$ around Greenland is more likely to be a product of an anomalous Rossby wavetrain, rather than its initiator. On the other hand, looking further afield, Fig. 6(a) does implicate Rossby wave source regions over the north Pacific whose sign is consistent with $\bar{\zeta}_{\text {a }}^{G}$, and which might be the initiator of the high-latitude wavetrain. There are also features over the central Atlantic whose sign is more ambiguous.

Figure 6(b) shows the contribution from anomalous transients. Although the global average effect is negative and weakly supports $\bar{\zeta}_{\mathrm{a}}^{G}$, local anomalous transients exert a marked positive effect, weakening the regional anomaly. Thus the strong effects of local transients are nullified by the effects of remote transients. Figure 6(c) shows the contribution from sources not described by the linear model. These effects cannot be interpreted in terms of linear wave propagation, and are indeed predominantly local to the target region: baroclinic and nonlinear effects act locally to support the anomaly. The damping term, $\alpha \xi_{\mathrm{a}} \zeta^{*}$, mirrors $\bar{\zeta}_{\mathrm{a}}^{G}$ and thus provides a trivial contribution. Clearly 

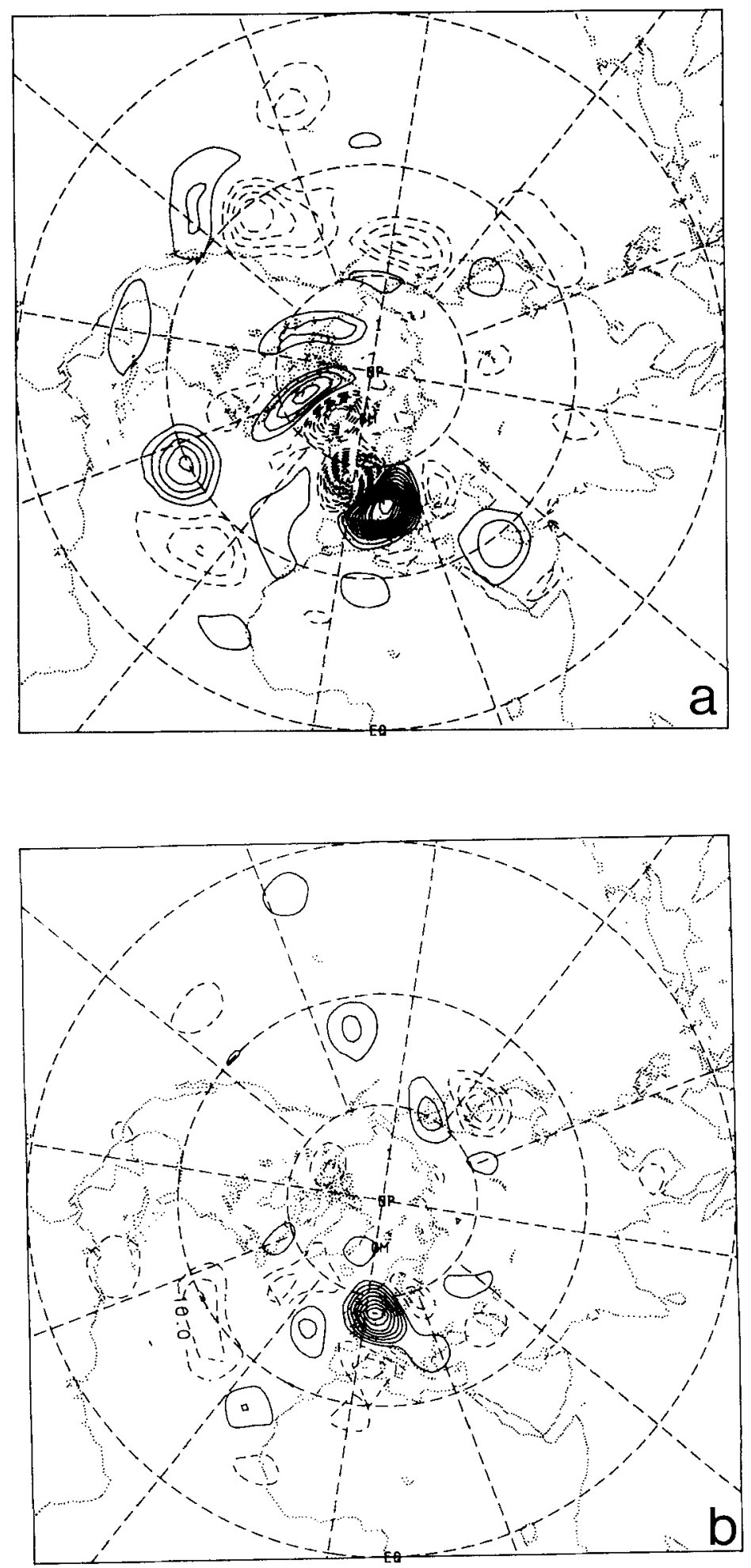

Figure 6. Distributions of (a) $R_{\mathrm{a}} \xi^{*}$; (b) $T_{\mathrm{a}} \zeta^{*}$; (c) $C \zeta^{*}$ for Case I. Contour interval $10 \mathrm{~s}^{-1} A^{-1}$; zero contour omitted. 


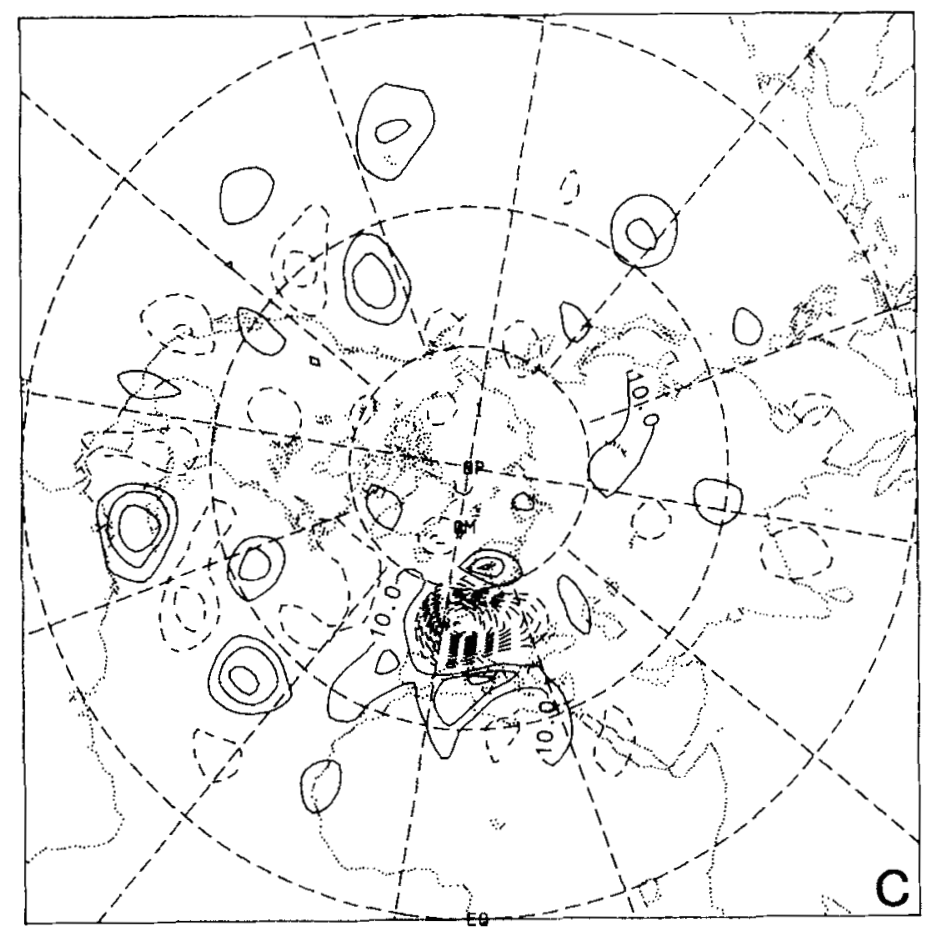

Figure 6. Continued.

for the method to be at all effective, this contribution to $\int C \zeta^{*}$ (given in brackets in Table 1) must be small. We investigate sensitivity to $\alpha$ in the next section.

Taking the remote Rossby wave sources selected by $\zeta^{*}$ to be the most interesting aspect of the analysis, it is interesting to speculate on their origins. Figure 7(a) illustrates the GCM's anomalous divergence at $300 \mathrm{mb}$, which provides the main component to $R_{\mathrm{a}}$ via $f \nabla \cdot \mathbf{v}_{\mathrm{a}}$. By comparing with $R_{\mathrm{a}} \zeta^{*}$, it would appear that the associated divergence anomalies over the north-east Pacific constitute part of an anomalous Walker-Hadley circulation spanning the Pacific. However, there is no simple link between this latter and the associated s.s.t. anomalies illustrated in Fig. 7(b).

\section{(b) Case 1: Sensitivity experiments}

(i) Waves in the basic state. To illustrate the role of zonal asymmetries in the basic state, the adjoint analysis in the previous section has been repeated using a zonally symmetric basic state; the results are tabulated in the second row of Table 1. Although the correction term is scarcely larger than in the wavy basic-state case, it conceals a very large damping contribution which cancels other model errors. The size of the damping term makes the analysis trivial in this case. The adjoint solution $\zeta^{*}$, plotted in Fig. 8, is radically different to the wavy case in Fig. 5. It consists primarily of an in situ response to $F^{*}$ (which has zero zonal mean so that there is weak negative forcing elsewhere in the latitude band of central Europe), with very little wave propagation. Thus although Table 1 might suggest that the effects of zonal asymmetries are largely accounted for by the large linear damping contribution, Fig. 8 strongly suggests that the result is trivial, with the local damping contribution $\alpha \zeta_{\mathrm{a}} \zeta^{*}$ accounting for $\bar{\zeta}_{\mathrm{a}}$; this is confirmed by the spatial field of the former quantity (not shown). 

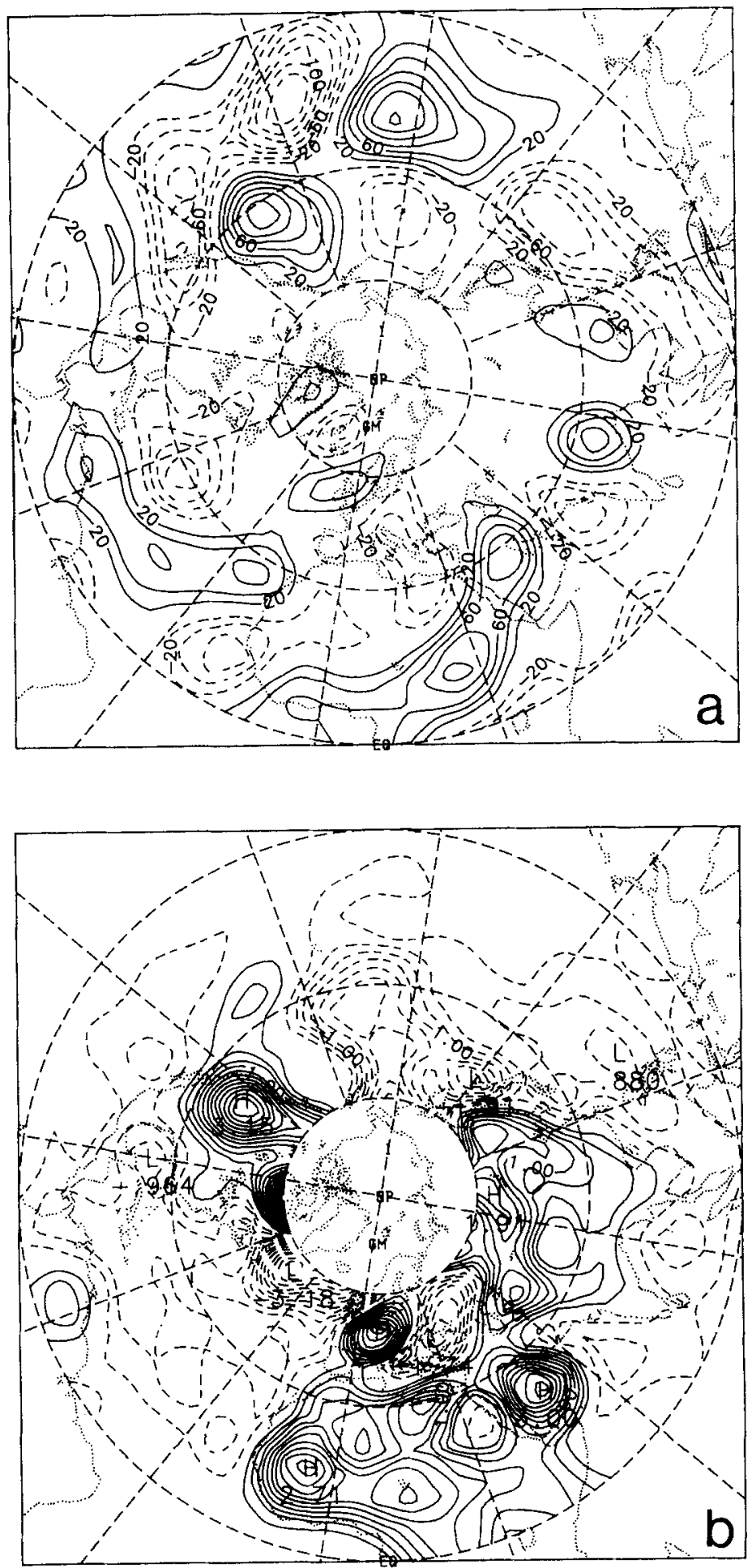

Figure 7. GCM anomalies of (a) $300 \mathrm{mb}$ divergence smoothed with a spectral filter (contour interval $20 \times 10^{-8} \mathrm{~s}^{-1}$ ); (b) surface temperature south of latitude $60^{\circ} \mathrm{N}$ (contour interval $0.25 \mathrm{~K}$ ). Both maps have the zonal mean removed and the zero contour omitted. 


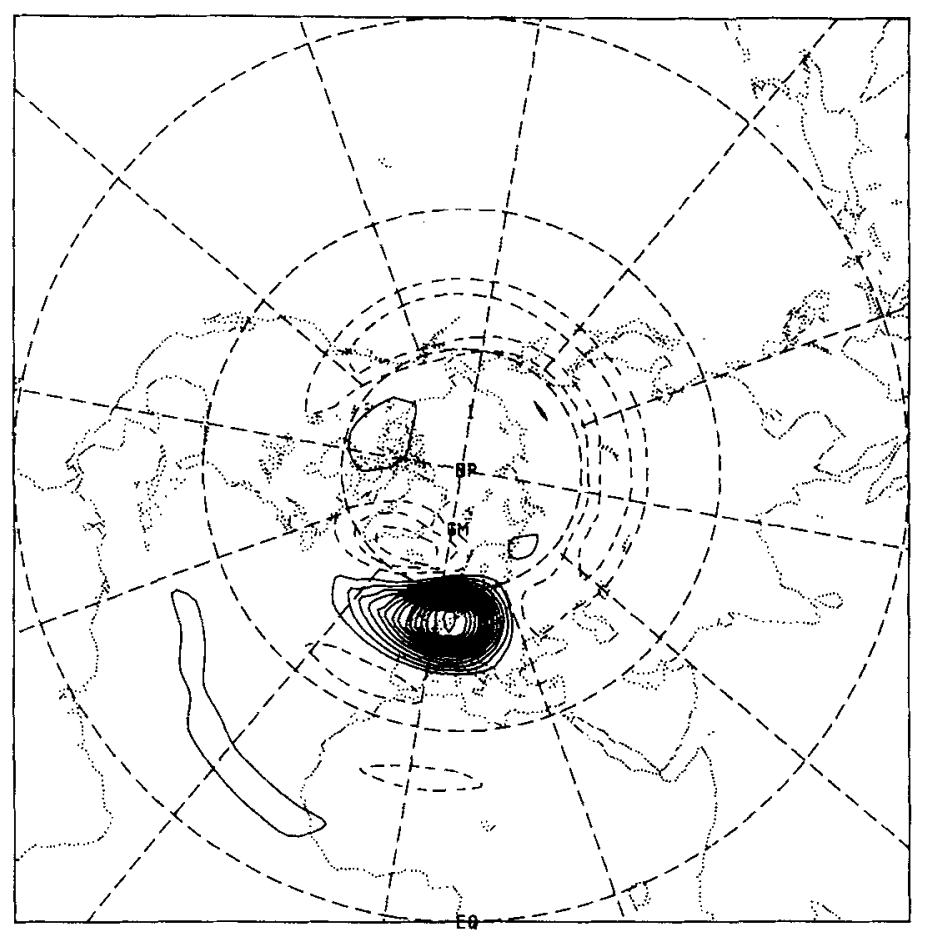

Figure 8. Adjoint solution $\zeta^{*}$ for Case I, linearizing about a zonally-symmetric basic state. Contour interval $25 \mathrm{~s}^{-1}$, zero contour omitted.

(ii) Linear damping. To demonstrate sensitivity to the linear damping, the third and fourth rows in Table 1 illustrate the effect of halving and doubling the damping strength on the wavy basic-state experiment, respectively. The correction term is very large in both cases, which means that the linear model is performing badly. Furthermore, in both cases the damping contribution is larger than for $\alpha^{-1}=5 \mathrm{~d}$, so the adjoint analysis includes a larger trivial component. At $\alpha^{-1}=2.5 \mathrm{~d}$, the damping contribution to $\bar{\zeta}_{\mathrm{a}}^{G}$ becomes dominant making the adjoint analysis largely trivial, even though the role of errors in the linear model (i.e. the remaining terms in (10)) is somewhat reduced. In contrast, halving the damping strength $(10 \mathrm{~d})^{-1}$ increases the effects of model errors. The choice of $\alpha^{-1}=5 \mathrm{~d}$ thus results in by far the smallest correction term, with the smallest trivial damping component.

Figure 9 illustrates $\zeta^{*}$ for $\alpha^{-1}=10 \mathrm{~d}$ (part (a)) and $\alpha^{-1}=2.5 \mathrm{~d}$ (part (b)). Halving the damping strength increases the influence of remote regions, especially low latitudes, whilst decreasing the influence of local forcing. Doubling the damping strength has the opposite effect, increasing the influence of local forcing at the expense of remote regions. The amplitude of the adjoint solution exhibits a more or less linear sensitivity to Rayleigh damping. Remote features of $\zeta^{*}$ are amplified at low damping, but they are also present in the case of high damping. On the other hand, the forced direct solution in Fig. 3(b) is much more sensitive, with the same 4-fold damping change leading to a 400 -fold change in solution amplitude, and a complete change in structure (not shown)! A major difference between adjoint and direct problems is that the latter is forced by $F_{\mathrm{a}}$, which is global, whilst the former is forced by $F^{*}$, which is local. Since it is the projection of the forcing on to the adjoint normal modes of the linear operator which determines which normal modes will be excited (Zhang 1988), a global forcing distribution should be capable of 

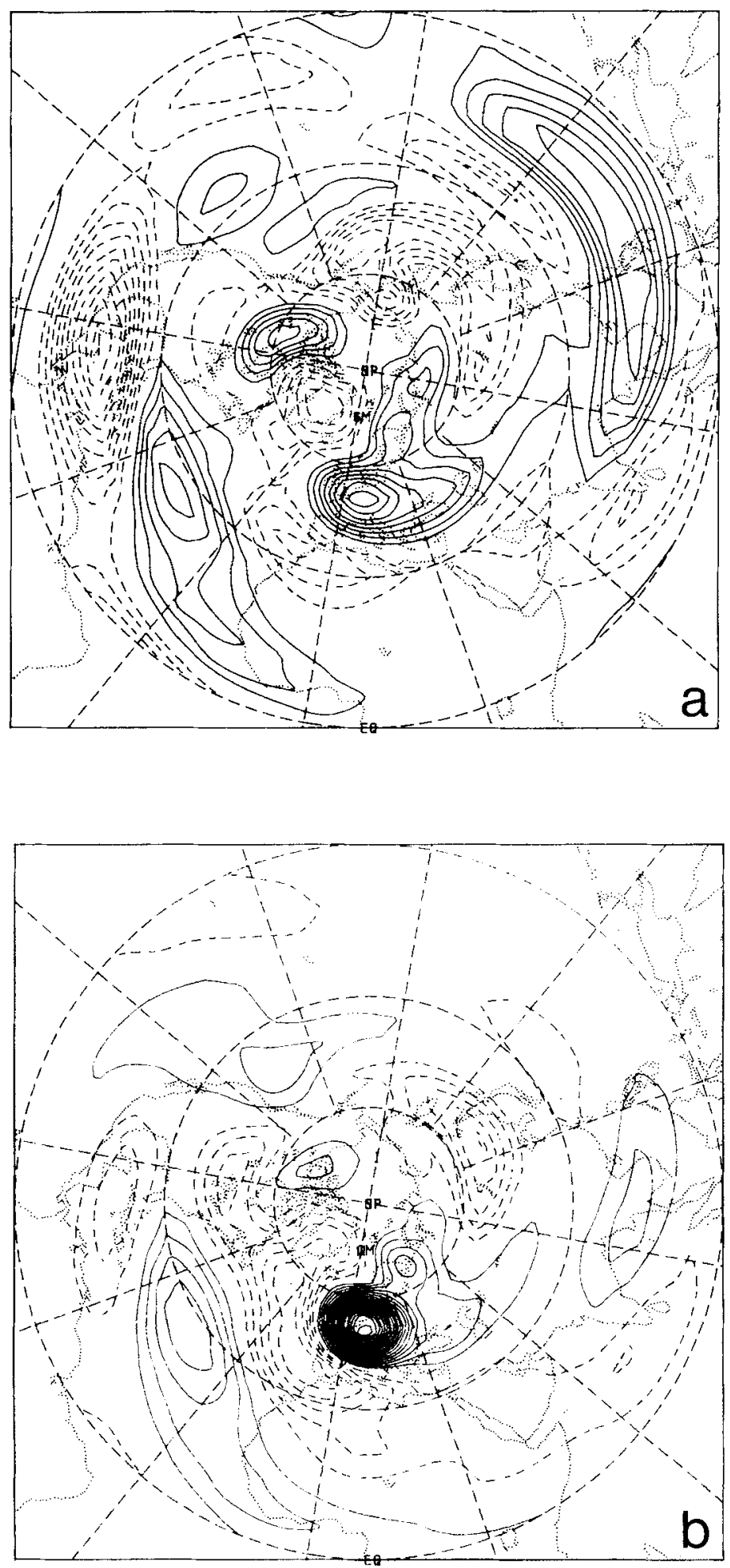

Figure 9. Sensitivity of $\zeta^{*}$ in Fig. 5 to linear damping: (a) $\alpha^{-1}=10 \mathrm{~d}$; (b) $\alpha^{-1}=2.5 \mathrm{~d}$. Contour intervals 50 and $12.5 \mathrm{~s} A^{-1}$ respectively; zero contour omitted. 
exciting many more modes than a local forcing. In the direct linear forced problem, the modes which dominate the solution are essentially selected by the choice of the damping. Thus, in the globally forced problem, we essentially select different near-resonant modes when we reduce the damping (cf. Branstator 1991). In the adjoint problem, on the other hand, $F^{*}$ presumably excites fewer normal modes (of the adjoint problem), while an increase in the damping strength in fact results in a more regionally confined smalleramplitude response.

Maps of $R_{\mathrm{a}} \zeta^{*}$ (not shown) still exhibit the basic structure seen in Fig. 6(a) in the above experiments (besides, $R$ does not change), with the distribution being merely more regionally confined in the strong damping case, but scarcely different (visually) in the weak damping case. This is quite at odds with the changes in the global integrals in Table 1 , and demonstrates the sensitivity to relatively small changes in $\zeta^{*}$. It is the effect of these small changes on $R_{\mathrm{a}}, T_{\mathrm{a}}$ and $C$ in Table 1 which mirror the large changes of the direct linear solution (to global forcing) over the target area. This makes interpretation of details of the main wavetrain structure in $R_{\mathrm{a}} \xi^{*}$ rather precarious.

(iii) Alternative basic state. In the above experiments the models were linearized about $\zeta_{c}$, the control basic state. However, as far as linear theory is concerned, it is just as legitimate to linearize about $\zeta_{\mathrm{r}}$, the $2 \times \mathrm{CO}_{2}$ basic state, and this can give us some indication on sensitivity. The bottom row of Table 1 presents the results of doing this; from which it can be seen that the sensitivity is quite large. Although the main results are qualitatively similar (and the associated spatial distributions, not shown, support this), anomalous Rossby wave sources now account for some $90 \%$ of the regional anomaly, with almost zero contribution from transients. The correction term is very small, but the effects of model errors are offset by the effects of the linear damping in this case. We conclude that the importance of anomalous Rossby wave sources is the only robust feature.

\section{(c) Cases II and III. Interdecadal variability}

In this section we present briefly the results of applying our adjoint analysis to two purely interdecadal central European anomalies defined as the difference between decades $\mathrm{A}$ and $\mathrm{B}$ in Fig. 1: $\zeta_{\mathrm{a}}=\frac{1}{2}\left(\zeta_{\mathrm{B}}-\zeta_{\mathrm{A}}\right)$, where the subscript refers to the respective decadal winter mean, in the control run (Case II) and the $2 \times \mathrm{CO}_{2}$ experiment (Case III), respectively. The results of the analyses using a 5-day damping are given in Table 2 , where, in each case, we linearize about a wavy basic state defined as $\bar{\zeta}=\frac{1}{2}\left(\zeta_{\mathrm{A}}+\right.$ $\left.\zeta_{\mathrm{B}}\right)$. The regional anomalies $\bar{\zeta}_{\mathrm{a}}^{G}$ are about half the amplitude of that in Case 1 , but, again, anomalous Rossby wave sources are the largest single contributor. In contrast to Case I, transients act to dissipate both anomalies. According to the size of the correction term, linear barotropic theory is particularly good in Case III, where the size of the damping term is very small indeed. In fact since we are linearizing about a basic state which is exactly midway between the flows defining the anomaly, the nonlinear term is

TABLE 2. ADJOINT DIAGNOSES OF CASES II AND III.

\begin{tabular}{lcccc}
\hline Experiment & $\begin{array}{c}\text { Vorticity anomaly } \\
\bar{\zeta}_{\mathrm{a}}\end{array}$ & $\begin{array}{c}\text { RWS } \\
\int R_{\mathrm{a}} \zeta^{*}\end{array}$ & $\begin{array}{c}\text { Transients } \\
\int T_{\mathrm{a}} \zeta^{*}\end{array}$ & $\begin{array}{c}\text { Correction } \\
\int C \zeta^{*}\left(\int \alpha \zeta^{*}\right)\end{array}$ \\
\hline Case II & -2.18 & -1.66 & +0.70 & $-1.22(-0.90)$ \\
Case III & +2.84 & +2.50 & -0.38 & $+0.72(+0.15)$ \\
\hline
\end{tabular}

Case II: control run, decade B minus A. Case III: $2 \times \mathrm{CO}_{2}$ run, decade B minus A. Wavy basic states: see text. Units are $10^{-6} \mathrm{~s}^{-1}$. 
identically zero (Held and Kang 1987), so that all errors are associated with the barotropic approximation. Table 1 indicates that there is considerable sensitivity to the choice of basic state, so that using $\bar{\zeta}=\zeta_{A}$, or $\bar{\zeta}=\zeta_{B}$ instead, might yield qualitatively different results.

The spatial distribution of $\zeta^{*}$ (not shown) is quite similar to Fig. 5 in both cases, although, as we have seen, small differences can be important. Figure 10 illustrates maps of $R_{\mathrm{a}} \zeta^{*}$-the dominant contribution in Table 2 . In both cases, the structure is quite similar to that in Case I (Fig. 6(a)), except for a change of polarity in Case III. Maps of $T \zeta^{*}$, (not shown) also resemble that of Case I, with significant influence of transient forcing locally in the target region itself, over the Atlantic and north of Japan. These local effects again act to weaken $\bar{\zeta}_{\mathrm{a}}^{G}$.

\section{Discussion}

In this paper we have used the locally-forced adjoint problem to analyse a regional feature of the usual globally forced (direct) linear barotropic problem. By using the latter to attempt to simulate interdecadal winter anomalies in GCM climate experiments, our motivation was to find aspects of the barotropic model's forcing distribution specifically implicated in the local solution over Europe. However, the main results relate primarily to the analysis technique itself and to barotropic linear theory, and only tentative conclusions can be drawn on the roots of the regional anomalies. Summarizing the main results, we have:

- The linear model was able to account for $45-75 \%$ of the three regional decadal anomalies studied in terms of anomalous Rossby wave sources and anomalous transients. The former always constituted the larger contribution, though its magnitude proved to be sensitive to the choice of the basic state. There was cancellation between the two physical sources of the anomaly in two out of the three cases-an inherent problem with this kind of linear analysis (cf. Branstator (1992)).

- We were able to identify regions where anomalous Rossby wave sources (associated with upper-level divergence anomalies) and vorticity tendencies associated with anomalous transients were directly implicated in the European vorticity anomaly. However, a pronounced spatial cancellation between such regions made it difficult to determine visually even the sign of the globally integrated effect.

- Anomalous forcing over the target region itself and the immediate vicinity upstream gave the largest contribution to the anomaly. The linear barotropic model was necessarily ineffective so far as the origins of the anomalous transients and the divergence, so implicated, were concerned. Nevertheless, some remote regions also were identified as being possible sources of Rossby waves; they themselves organizing the local forcing.

- Rossby wave sources over the east and north Pacific, and over the central Atlantic were identified as important. In the present case no accompanying s.s.t. anomalies could be identified and a linearized primitive-equation model forced by anomalous heating instead of upper-level divergence might clarify the issue.

- The structure of the locally forced adjoint mode turned out to be considerably more robust than its globally-forced direct counterpart, both with respect to the basic state and to Rayleigh damping. Nevertheless, details of the adjoint mode's structure proved to be important in accounting for $\bar{\zeta}^{G}$, so that, as one might expect, the sensitivity inherent in the linear model cannot be circumvented by simply considering its adjoint. 

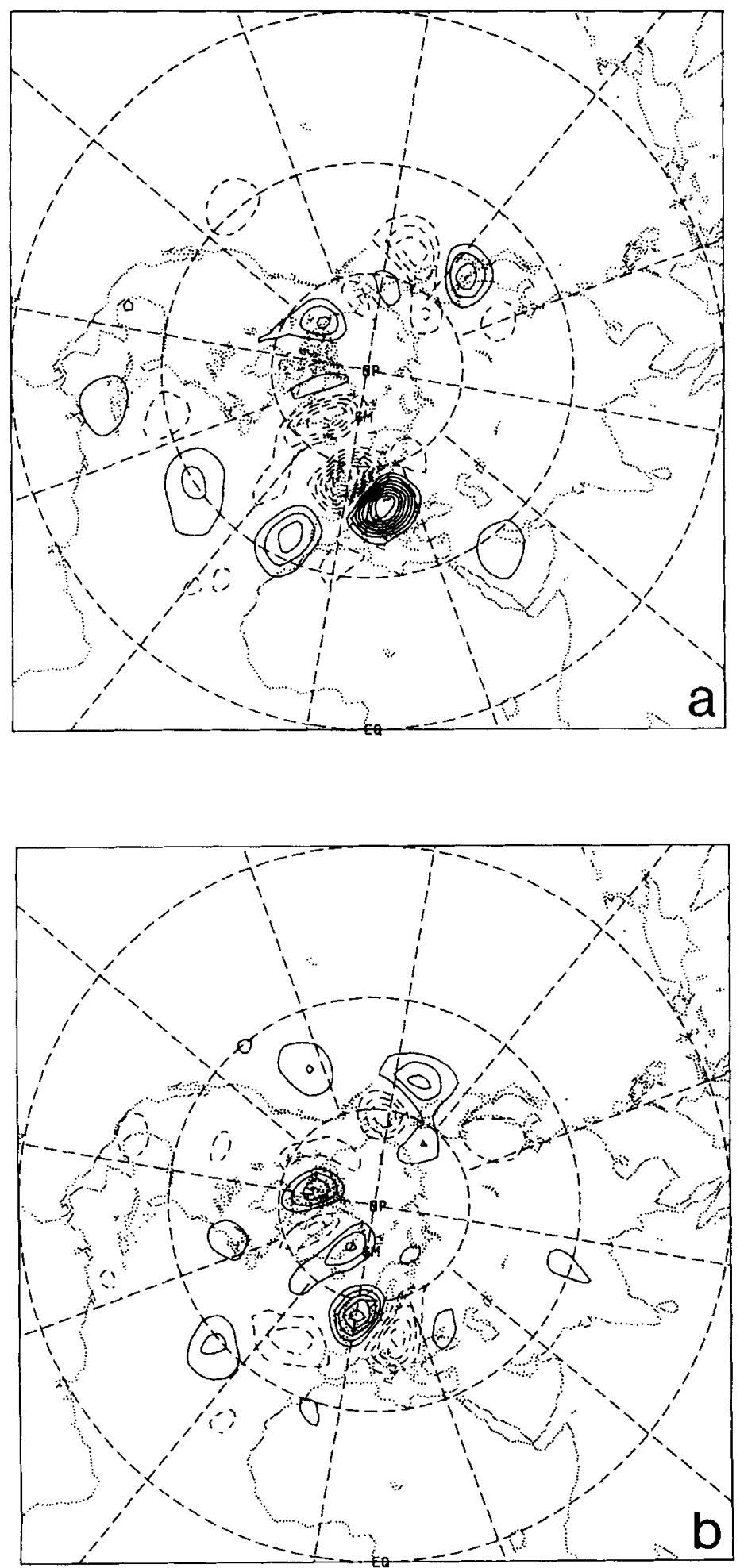

Figure 10. Distributions of $R_{\mathrm{a}} \zeta^{*}$ : (a) Case II; (b) Case III. Contour interval $10 \mathrm{~s}^{-1} A^{-1}$; zero contour omitted. 
- The correct choice of linear damping proved to be crucial to success in applying the adjoint method to the linear barotropic model; this mirrors the sensitivity of the globallyforced direct linear problem to damping. With too much damping, the method became trivial and over regionalized, whilst with too little, errors associated with baroclinic and nonlinear effects assumed greater importance.

- The applicability of the linear barotropic model to interdecadal vorticity anomalies is limited. In particular, its performance here does not match up to the results of Held and Kang (1987) for the extra-tropical response to El Niño. We have used a steady-state externally forced atmosphere (e.g. by the ocean) to model interdecadal anomalies. However, the atmosphere itself may be capable of generating interdecadal variability, as suggested by James and James (1989), and complex interactions between the atmosphere and ocean may make the steady-atmosphere hypothesis questionable.

In order to check the relevance of our three case studies to the entire GCM experiments, we have constructed one-point correlation maps for each GCM winter time series, with a point over central Europe as base point. Figure 11 illustrates such maps
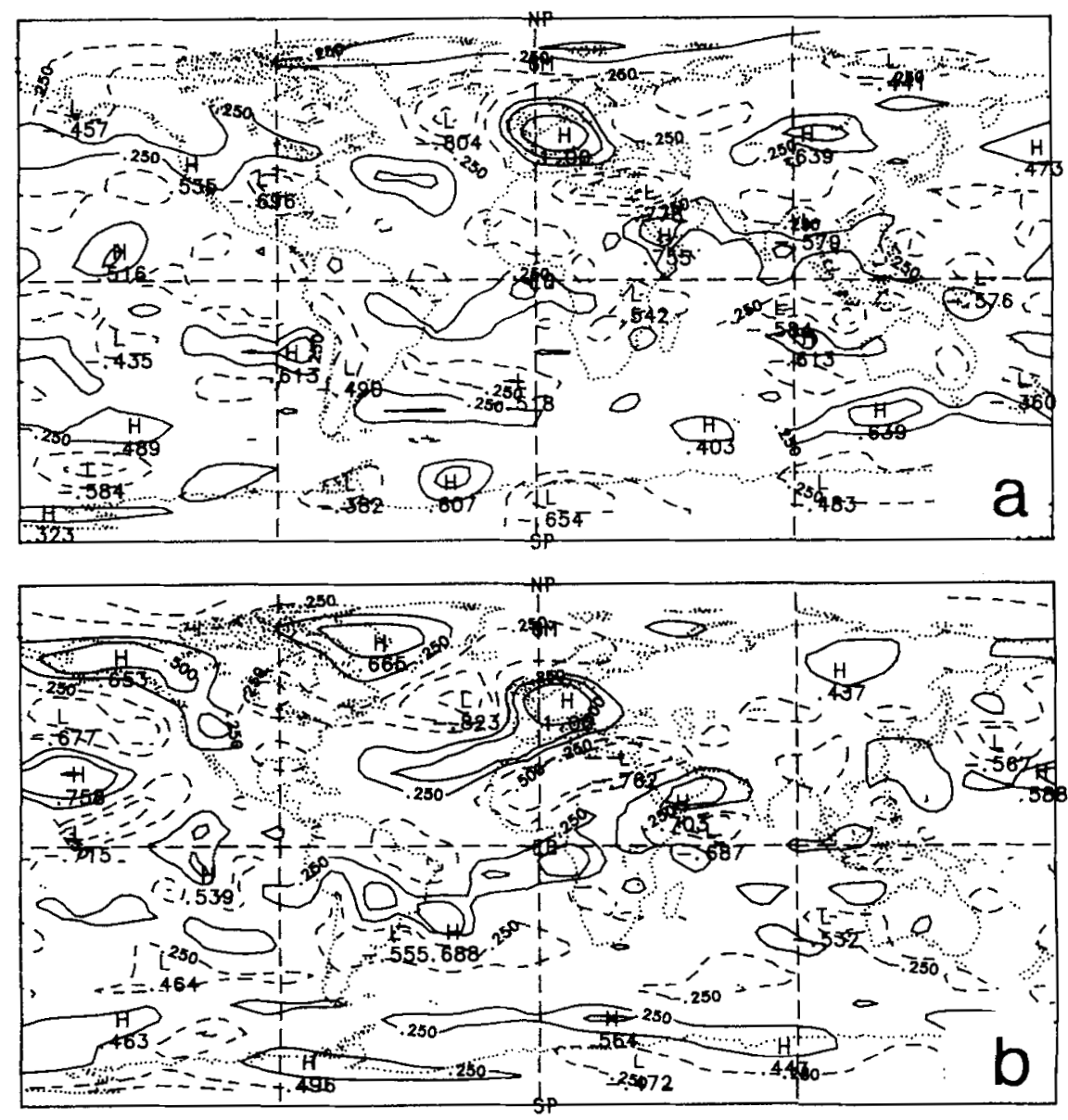

Figure 11. One-point correlation maps of low-pass filtered 99-winter $300 \mathrm{mb}$ vorticity time series (from which the time mean was first substracted). Base point is at position $50^{\circ} \mathrm{N}, 10^{\circ} \mathrm{E}$ (near Würzburg): (a) control; (b) $2 \times \mathrm{CO}_{2}$ GCM experiments. Contour interval 0.25 ; zero contour omitted. The $95 \%$ significance level is at 0.58 (10 degrees of freedom) and significant correlations cover approximately (a) $3 \%$ and (b) $6 \%$ of the area of the northern hemisphere. 
for the low-pass (periods greater than about 10 winters) filtered $300 \mathrm{mb}$ vorticity time series, from which it transpires that the main wave-train structure from the east Pacific across to Europe, found in Case $\mathrm{I}\left(2 \times \mathrm{CO}_{2}-\right.$ control), is a recurring pattern in the $2 \times \mathrm{CO}_{2}$ experiment, but not in the control. This could well be connected with the better performance of linear theory in Case III compared with Case II. Thus, although the interdecadal variance is only slightly greater in the $2 \times \mathrm{CO}_{2}$ experiment than in the control, it does seem to be associated with a more coherent spatial structure in the $2 \times \mathrm{CO}_{2}$ run; indeed with a structure more amenable to linear analysis according to our experiments.

Linear theory suggests the importance of anomalous Rossby wave sources over the north-east Pacific for central European vorticity anomalies, at least in Cases I and III (Figs. 6(a) and 10(b)). To examine whether or not there is such a link, in a statistical sense over the entire low-pass winter time series, we plot in Fig. 12 one-point crosscorrelation maps of the $300 \mathrm{mb}$ vorticity at our central European basepoint with the Rossby wave source everywhere else. The contour $(0.58)$ indicates the threshold of local statistical significance at the $95 \%$ level using 10 time degrees of freedom. Consistent with Fig. 11, remote correlations are generally higher in the $2 \times \mathrm{CO}_{2}$ run (Fig. 12(b)) and do indeed suggest a link with the north-east Pacific. The other noticeable regions of significant correlations lie slightly upstream of the basepoint, again anticipated by linear theory; although surprisingly there are no significant correlations over the basepoint itself or over Greenland.

The correlations in Fig. 12 (and indeed also in Fig. 11) are far from constituting globally significant patterns since locally significant correlations cover at most only about $6 \%$ of the area of the northern hemisphere (Fig. 11(b)). With 15 spatial degrees of freedom (determined as the $95 \%$ variance truncation point of principal component expansions of the lowpass time series (Metz 1991)), Livezey and Chen (1983) indicate that at least about $20 \%$ areal coverage is required for global significance.

The above cross-correlation analysis was repeated using s.s.t./ground temperature in place of the Rossby wave source (not shown). A surprisingly coherent cross-correlation pattern over the Pacific/North American sector (maximum correlation 0.75; approx. 7\% area locally significant) was found in the $2 \times \mathrm{CO}_{2}$ time series, whilst no pattern emerged from the control time series (approx. 3\% area locally significant). This is consistent with Fig. 12 and suggests that variations in s.s.t. over the east Pacific may indeed be implicated in interdecadal European anomalies in the $2 \times \mathrm{CO}_{2}$ experiment. Our analysis suggests that application of the adjoint analysis to a primitive-equations model might further enlighten this process. The question of the absence of the phenomena from the control run remains to be answered.

\section{ACKNOWLEDGEMENTS}

I am indebted to W. Metz for the use of many of his computer programs and also thank him along with J. Egger, F. Schmidt and V. Wirth for reading a draft of the manuscript and for many stimulating discussions. I am grateful to the Hamburg climate group at the Meteorological Institute of the University of Hamburg and the Max Planck Institute for Meteorology for providing the model data, and for their assistance. This work was supported by the Bavarian climate program (BayFORKLIM) and by the German Federal Ministry for Research and Technology (BMFT).

\section{APPENDIX}

Vorticity is expressed as a series of spherical harmonic coefficients truncated triangularly at total wavenumber $N=21$-the resolution of the GCM. In terms of these 

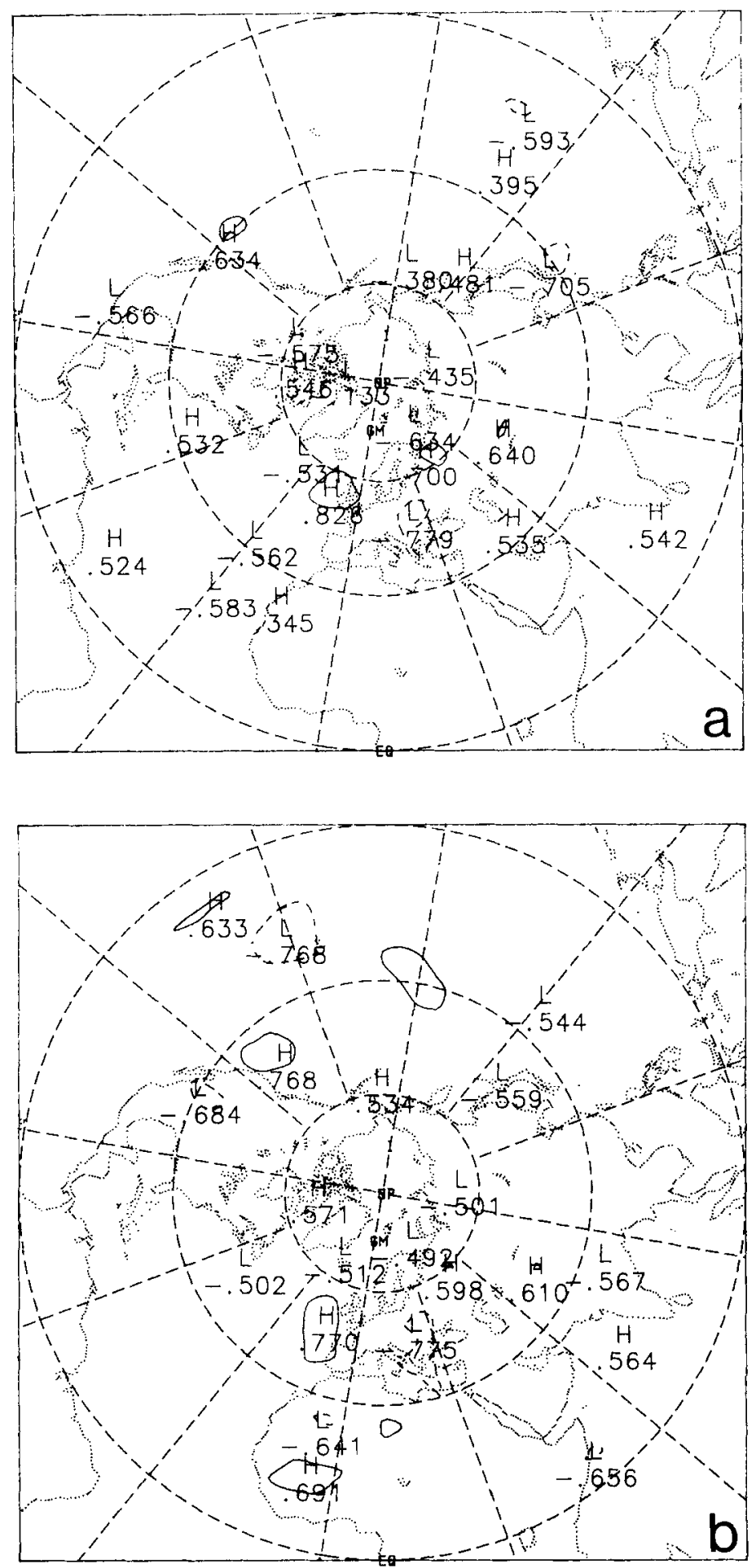

Figure 12. The correlation of the $300 \mathrm{mb}$ vorticity at position $50^{\circ} \mathrm{N}, 10^{\circ} \mathrm{E}$ with $R$ at all other points for lowpass filtered 99-winter time series with time mean substracted: (a) control; (b) $2 \times \mathrm{CO}_{2}$. The contour is at 0.58 which is the $95 \%$ significance level, with 10 degrees of freedom; significant correlations cover approximately (a) $3 \%$ and (b) $4 \%$ of the area of the northern hemisphere. 
spherical harmonics, the inner product (5) is given by the equations

$\langle\zeta, \boldsymbol{\eta}\rangle \equiv \frac{1}{4 \pi} \int_{-1}^{+1} \int_{0}^{2 \pi} \zeta \eta \mathrm{d} \lambda \mathrm{d} \mu=\frac{1}{4 \pi} \int_{-1}^{+1} \int_{0}^{2 \pi}\left(\sum_{j}^{N} \zeta_{j} Y_{j}\right)\left(\sum_{k}^{N} \eta_{k} Y_{k}\right) \mathrm{d} \lambda \mathrm{d} \mu$

where $Y_{j}$ are the spherical harmonics $Y_{n_{j}}^{m_{j}}$. Using the orthogonality property

$$
\frac{1}{4 \pi} \int_{-1}^{+1} \int_{0}^{2 \pi} Y_{j} Y_{k}^{*} \mathrm{~d} \lambda \mathrm{d} \mu=\delta_{j k}
$$

we obtain

$$
\langle\boldsymbol{\zeta}, \boldsymbol{\eta}\rangle=\sum_{j=-N}^{N} \zeta_{j} \eta_{j}^{*}=\sum_{\substack{j=1 \\ m_{j}=0}}^{N} \zeta_{j} \eta_{j}^{*}+2 \operatorname{Re}\left\{\sum_{\substack{j=1 \\ m_{j} \neq 0}}^{N} \zeta_{j} \eta_{j}^{*}\right\}
$$

In vector notation we have

$$
\langle\boldsymbol{\zeta}, \boldsymbol{\eta}\rangle=\operatorname{Re}\left\{\boldsymbol{\zeta}^{\mathrm{T}} \mathbf{D} \boldsymbol{\eta}\right\}
$$

where $\mathrm{T}$ denotes the transpose conjugate, $\zeta^{\mathrm{T}}=\left(\zeta_{1}^{*}, \ldots, \zeta_{N}^{*}\right)$ etc, and $\mathbf{D}$ is a diagonal matrix whose diagonal entries are 1 for $m=0$ and 2 for $m \neq 0$. Zhang (1988) has shown that the discretized inner product under energy and streamfunction-squared norms also takes the form (A3) if $\mathbf{D}$ is chosen appropriately.

If $\mathbf{A}$ is the (real) matrix of the differential operator and $\mathbf{A}^{*}$ is its adjoint, then under (A3), the Green's identity is

$$
\operatorname{Re}\left\{(\mathbf{A} \zeta)^{\mathrm{T}} \mathbf{D} \boldsymbol{\eta}\right\}=\operatorname{Re}\left\{\boldsymbol{\zeta}^{\mathrm{T}} \mathbf{D}\left(\mathbf{A}^{*} \boldsymbol{\eta}\right)\right\}
$$

so that

$$
\mathbf{A}^{*}=\mathbf{D}^{-1} \mathbf{A}^{\mathrm{T}} \mathbf{D}
$$

defines the adjoint which is essentially the transpose of $\mathbf{A}$ under the enstrophy norm.

To see how the adjoint method depends on the norm chosen, the discretized form of (4) can be written

$$
\boldsymbol{\zeta}^{\mathrm{T}} \mathbf{D F} \mathbf{F}^{*}=\boldsymbol{\zeta}^{* \mathrm{~T}} \mathbf{D F}
$$

Using (A5) the discretized adjoint problem (3) reads

$$
\mathbf{D}^{-1} \mathbf{A}^{\mathrm{T}} \mathbf{D} \zeta^{*}=\mathbf{F}^{*}
$$

so that

$$
\zeta^{*}=\mathbf{D}^{-1}\left(\mathbf{A}^{\mathrm{T}}\right)^{-1}\left(\mathbf{D F}^{*}\right)
$$

Substituting (A8) into (A6) and rearranging yields

$$
\zeta^{\mathrm{T}}\left(\mathbf{D F} \mathbf{F}^{*}\right)=\left\{\left(\mathbf{A}^{\mathrm{T}}\right)^{-1}\left(\mathbf{D} \mathbf{F}^{*}\right)\right\}^{\mathrm{T}} \mathbf{F} .
$$

Equation (A6) indicates that $\mathbf{D F}^{*}$ represents the weighting function defining the regional vorticity anomaly, whilst from (A8) it also defines the forcing function in the adjoint equation. Under the enstrophy norm $\mathbf{D}$ is essentially the identity matrix, whilst under the energy and streamfunction-squared norms $\mathbf{D}$ represents the inverse Laplacian and the inverse Laplacian squared, respectively (see Zhang 1988). Thus for the same $\mathbf{F}^{*}$, $\mathbf{D F}^{*}$ becomes increasingly spread out under the latter norms. However, if we change the norm but keep $\mathbf{D F}^{*}$ invariant, the choice of norm will be irrelevant (cf. (A9)). In 
conclusion, the enstrophy norm yields the simplest formulation of the adjoint method and makes the obvious choice.

\author{
Branstator, G. \\ Cubasch, U., Hasselmann, K., \\ Höck, H., Maier-Reimer, E., \\ Mikolajewicz, U., Santer, B. D. \\ and Sausen R. \\ Hasselmann, K
}

Held, I. M. and Kang, I.-S.

Hoskins, B. J. and Karoly, D. J.

James, I. N. and James, P. M.

Livezey, R. E. and Chen, W. Y.

Marchuk, G. I.

Mikolajewicz, U. and

Maier-Reimer, E.

Metz, W.

Robertson, A. W.

Zhang, Z

\section{REFERENCES}

1991 The maintenance of low-frequency atmospheric anomalies. $J$. Atmos. Sci., 49, 1924-1945

1991 Transient greenhouse warming computations with a coupled ocean-atmosphere model. Report No. 67, Max Planck Institute for Meteorology, Hamburg

1976 Stochastic climate models. Part I: Theory. Tellus, 28, 473-485

1987 Barotropic models of the extratropical response to El Niño. $J$. Atmos. Sci. 44, 3576-3586

1981 The steady linear response of a spherical atmosphere to thermal and orographic forcing. J. Atmos. Sci., 38, 1179-1196

1989 Ultra-low-frequency variability in a simple atmospheric circulation model. Nature, 342, 53-55

1983 Statistical field significance and its determination by Monte Carlo techniques, Mon. Weather Rev., 111, 46-59

Numerical solutions of problems in atmospheric and oceanic dynamics. Gidrometeoizdat Press (in Russian)

1990 Internal secular variability in an ocean general circulation model. Clim. Dyn., 4, 145-156

1991 Optimal relationship of large-scale flow patterns and the barotropic feedback due to high-frequency eddies. J. Atmos. Sci., 48, 1141-1159

1992a Diagnosis of regional monthly anomalies using the adjoint method. Part I: Temperature. J. Atmos. Sci., 49, 885905

1992b An examination of simulated regional climate change using the adjoint method. In Studying climate with the atmospheric model ECHAM. Ed. R. Sausen. Large-scale atmospheric modelling, Report No. 1, Meteorological Institute University of Hamburg, FRG

1988
'The linear study of zonally asymmetric barotropic flows'. $\mathrm{Ph}$. D. thesis, University of Reading. (Obtainable from The British Library, Boston Spa LS23 7BQ). 